\title{
Smart Helmet with Emergency Notification System-A Prototype
}

\author{
S. Sasirekha, I. Joe Louis Paul \\ Department of Information Technology, SSN College of \\ Engineering, Chennai, Tamil Nadu, India \\ E-mail: sasirekhas@ssn.edu.in, joelouisi@ssn.edu.in
}

\author{
S. Swamynathan \\ Department of Information Science and Technology, Anna \\ University, Chennai, Tamil Nadu, India \\ E-mail: swamyns@annauniv.edu
}

\author{
Gokul. Y, Kirthana.P \\ Department of Information Technology, SSN College of Engineering, Chennai, Tamil Nadu, India
}

\begin{abstract}
Motorcycles have higher rates of fatal accidents than any other automobiles in India. Hence, wearing a helmet alone is not sufficient to prevent accidents and save victims. Helmet wearing alone is not sufficient to save the life of a motor cyclist. The solution proposed in this work to address this life-threatening problem, is the Smart Helmet with Emergency Notification System (SHENS). This helmet not only protects the head of its rider but also his/her life by alerting the emergency service or an emergency contact when the rider has met with an accident. This smart helmet has the potential to act independently without any human intervention during road accidents. With the help of advanced sensors such as an infra-red sensor, impact sensor and accelerometer sensor, this smart helmet is also capable of detecting the consciousness of the accident victim and indicating the precise location of the accident. The alert to the emergency service is sent with the help of Global System for Mobile (GSM) module as a Short Messaging Service (SMS) containing the Global Positioning System (GPS) coordinates which indicate the location of the accident to which the emergency team is expected to reach to offer medical assistance. Thus, this smart helmet can be used to save millions and millions of lives by helping them to get timely medical attention in case of accidents.
\end{abstract}

Keywords-smart helmet; smart helmet with emergency notification system (SHENS); global system for mobile (GSM); global positioning system (GPS); accident notification; emergency notification

\section{INTRODUCTION}

The increase in population is the cause of many socio-economic problems and is the biggest challenge for the commuters worldwide. Being home of one-sixth of the human population, road travel in India is tedious and tiresome. In the current run, India has about 19.75 million vehicles registered [1] which accounts for the heavy and congested traffic on the roads. Due to this heavy count and poor road conditions, accidents tend to happen every day. It has been recorded that everyday about 400 people die in road accidents in India. Another important factor influencing the accident death rate is the lack of social responsibility of an individual. The proposed system aims to tackle all the problems as mentioned above by acting autonomously during the accident and by serving as a helping hand to the accident victim, ultimately, helping the victim to get swift and timely medical assistance.
Internet of Things (IoT) is emerging as a boon to humankind by offering a vast variety of reliable services using the embedded hardware, software and data processing [2]. The proposed smart helmet with the emergency notification system is also an IoT solution to eliminate the loss of lives due to road accidents. This proposed system, being powered by sensor networks and communication module that handles various data intelligently before executing a specified module. The algorithm depicted in the implementation section explains how the system reacts during the times of road accident, thus highlighting how this system can be a promising IoT solution to prevent the loss of lives in accidents. Moreover, this proposed system can also become a day to day gadget like cell phones since it has the greatest scope and importance of saving a person's life during road accidents.

In the remainder of the paper, Section II briefs about some of the related works carried out. In Section III, the system model of SHENS is illustrated. Then in Section IV, the implementation steps are discussed and in Section V, the test results obtained, and their inferences are elaborated. Finally, Section VI concludes.

\section{RELATED WORKS}

Several studies are being carried out in this crucial era of road accidents to help the victims or to create an accident-free road travel totally. Some of them are discussed below. The research work to enhance the emergency notification during accidents using machine-to-machine (M2M) technologies [3], the accident notification to the emergency service is carried out with the help of M2M technology without any human intervention. When an accident occurs, the system proposed in [3] categorizes the severity of the accident that occurred, into major, minor and moderate according to the motion of the sensor. The speed of the vehicle is determined with the help of Global Positioning System (GPS) module. However, the system in [3] has certain limitations. The accelerometer sensor is mounted on the surface of the vehicle, and so the severity of the condition of the accident victim is not taken into consideration. Since it has the cloud integration, data services, and Internet connectivity is very much needed for this system, thereby, it may result in latency to forward the message in the areas of weak reception. 
Likewise, the research work in [4] deals with the concept of determining the severity of the accident depending on the severity of the vehicle. The impact and pressure sensors are embedded on the surface detect the impact of the hit. Based on this, accident notification is sent to the emergency service. The severity of the accident is calculated using the concept of data mining by estimating the impact of the accident, determining the status of airbags and arriving at a theoretical prediction that an accident has occurred. The medical assistance can be sought immediately using this approach. The study of Wang Wei and Fan Hanbo in [5] aims at providing accident notification in case of car accidents. The vibration sensors embedded on the vehicle senses the shock during the accident and alerts the emergency service using the Global System for Mobile (GSM) module with the GPS coordinates.

Therefore, it is understood from the above illustration that many works are carried out to design a system which helps in times of emergency. Especially, with the advancement in the new advent technology, IoT, it has gained more attention [6-10]. Hence, in this work a Smart Helmet with Emergency Notification System (SHENS) based on IoT solution is proposed. The proposed system also uses GSM module for communication than cloud computing methodologies which are used in the works of [2] and [3], since mobile data or the Internet may not always be available on the roads.

\section{SYSTEM MODEL FOR SHNES}

The smart helmet contains a network of various sensors and modules embedded in it. The infra-red sensor, impact sensor, accelerometer sensor, the GPS module and a GSM are the main components of this Smart Helmet with Emergency Notification System (SHENS). The infra-red sensor is embedded inside the helmet to ensure that the person is wearing the helmet and only after that the entire helmet gets activated. The infra-red sensor also prevents the helmet from getting activated, when the helmet gets dropped accidentally. Next, the impact sensor is employed to determine the amount of impact with which the helmet hits the ground during the accident. When this impact crosses the particular threshold, the determination of which is explained in Section IV, the accelerometer sensor is employed to see if any head movements are found on the victim. In the meantime, the person can safely turn the system off if the emergency medical attention is not needed. If the victim is injured severely, the GPS module senses the exact location of the accident and the details of the location are sent as an SMS to the emergency medical service with the help of GSM module.

The proposed system is componentized into various modules in order to provide effective and immediate notification in case of accidents. The various processes that take place in SHENS are given as follows: Step 1: Helmet activation using the infra-red sensor, Step 2: Impact determination using impact sensor, Step 3: Consciousness detection using accelerometer, Step 4: Determination of location of the accident and Step 5: Notification to the emergency service.

The interrelationship among these modules can be well understood by the flow diagram which emphasizes the series of processes that take place within the SHENS system, as given in Fig. 1.

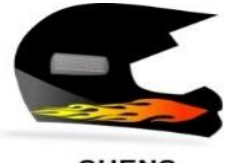

SHENS
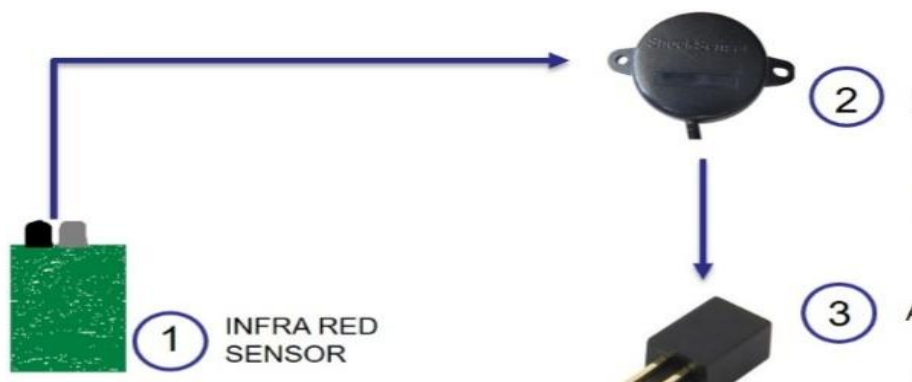

IMPACT SENSOR

Measures the impact of the hit and activates the accelerometer sensor.

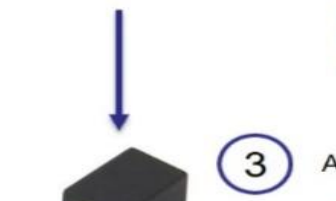

ACCELEROMETER

Determines the person's consciousness with the help of head movements. wearing the helmet or not.
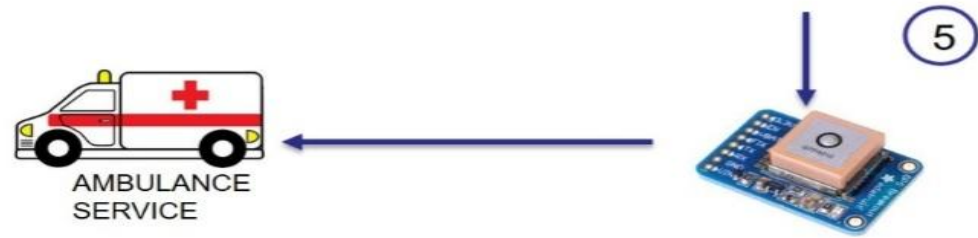

After the timer expires, the GPS coordinates and the emergency notification to the emergency service are sent through GSM module

Figure 1. System model of SHENS 


\section{A. Helmet Activation using Infra-Red Sensor}

The obstacle detection of the infra-red sensor [11] is the main principle involved in this module. The infra-red sensor consists of an infra-red transmitter and a receiver. In the case of any obstacle present in the path of the infra-red rays, they get reflected back to the receiver ensuring the presence of an obstacle. This same principle is applied for determining whether the helmet is worn by the rider or not. Hence, the infra-red sensor is mounted on the interior surface of the helmet. If the rider is wearing the helmet, then the infra-red sensor detects the constant obstacle which is the head of the rider within the helmet and keeps the entire system running. If the rider removes the helmet, infra-red sensor confirms that the person is not wearing the helmet and makes the smart helmet inactive. It comes in handy in various cases where the helmet falls accidentally causing fault notifications to the emergency service, which is a waste of time and resources. This system is very critical for the ideal functioning of SHENS.

\section{B. Impact Determination using Impact Sensor}

The impact sensor [12] is employed in SHENS to estimate the amount of impact on the surface of the helmet. It is very crucial for confirming that an accident has occurred. The impact sensor detects the sudden impact on the surface of the helmet. After the accident has occurred, producing an impact greater than the set threshold which is determined by using the equations given in the implementation section, while the accelerometer senses the tilt angles of the head [13].

\section{Consciousness Detection using Accelerometer}

The accelerometer sensor [14] senses a series of tilt angles after the person is met with an accident. The determination of tilt angles from the accelerometer sensor readings is described in Section IV. If the tilt angles are the same for a particular span of time, say 10 seconds, the accident victim is assumed to be not having any head movements. This lack of head movements is considered in this system as the victim is unconscious. If the tilt angles vary rapidly, the person is assumed to be conscious since head movements are present.

The timer module is one of the crucial components in SHENS responsible for setting the timer within the expiry of which the notification to the emergency service is sent. The timer module employed in SHENS is a software triggered inbuilt timer present on the Arduino Uno [15]. The timer can be visualized using a seven segment Light-Emitting Diode (LED) display which displays the countdown timer. The timer is set to two different intervals because there are two different scenarios of the person met with an accident being conscious or unconscious.
The two scenarios expected to be are as follows: If the accelerometer readings ensure that the person is conscious, the timer is set to 90 seconds. In this interval, the emergency notification can be aborted if no immediate medical assistance is needed. If the accelerometer readings show that the person is unconscious, the timer is set to 30 seconds. After the expiry of the timer, the determination of location phase is initiated.

\section{Determination of Location of the Accident}

The GPS module [16] comes into action immediately after the timer expires. The GPS module used in SHENS comes with a small form factor with an antenna capable of sending the GPS coordinates of the location in no time. The exact location of the person in the accident zone is determined in the form of latitude and longitude coordinates. These coordinates, upon decryption, provide the exact address of the accident zone for the emergency service to reach on time. The GPS module embedded in the SHENS is Skylab GPS module with a wired antenna for faster response of GPS coordinates from the satellite.

\section{E. Notification to the Emergency Service}

To send message or notification to the emergency service, a GSM module is embedded on the inner surface of the helmet. Upon successful discovery of the location of the accident, GSM module [17] comes into action. The GSM module takes a Subscriber Identification Module (SIM) to send the message to the emergency service. Very soon after GPS module identifies the location coordinates, the message in the format containing the GPS coordinates of the accident location is sent to the emergency service, thereby helping the medical service to reach the accident zone on time. Thus, providing accident notification on time along with the GPS coordinates of the location to emergency service can save millions of lives every day.

\section{IMPLEMENTATION}

The implementation of the Smart Helmet with Emergency Notification System (SHENS) is carried out in a series of steps that are designed algorithmically to carry out all of its features. The detailed description of the techniques and tools used is discussed below to get the better insight about the entire development process of this smart helmet.

\section{A. SHENS Working Algorithm}

The working mechanism of SHENS can be better understood with the help of algorithmic description given below. All the components are interfaced and programmed to function based on Algorithm 1. 


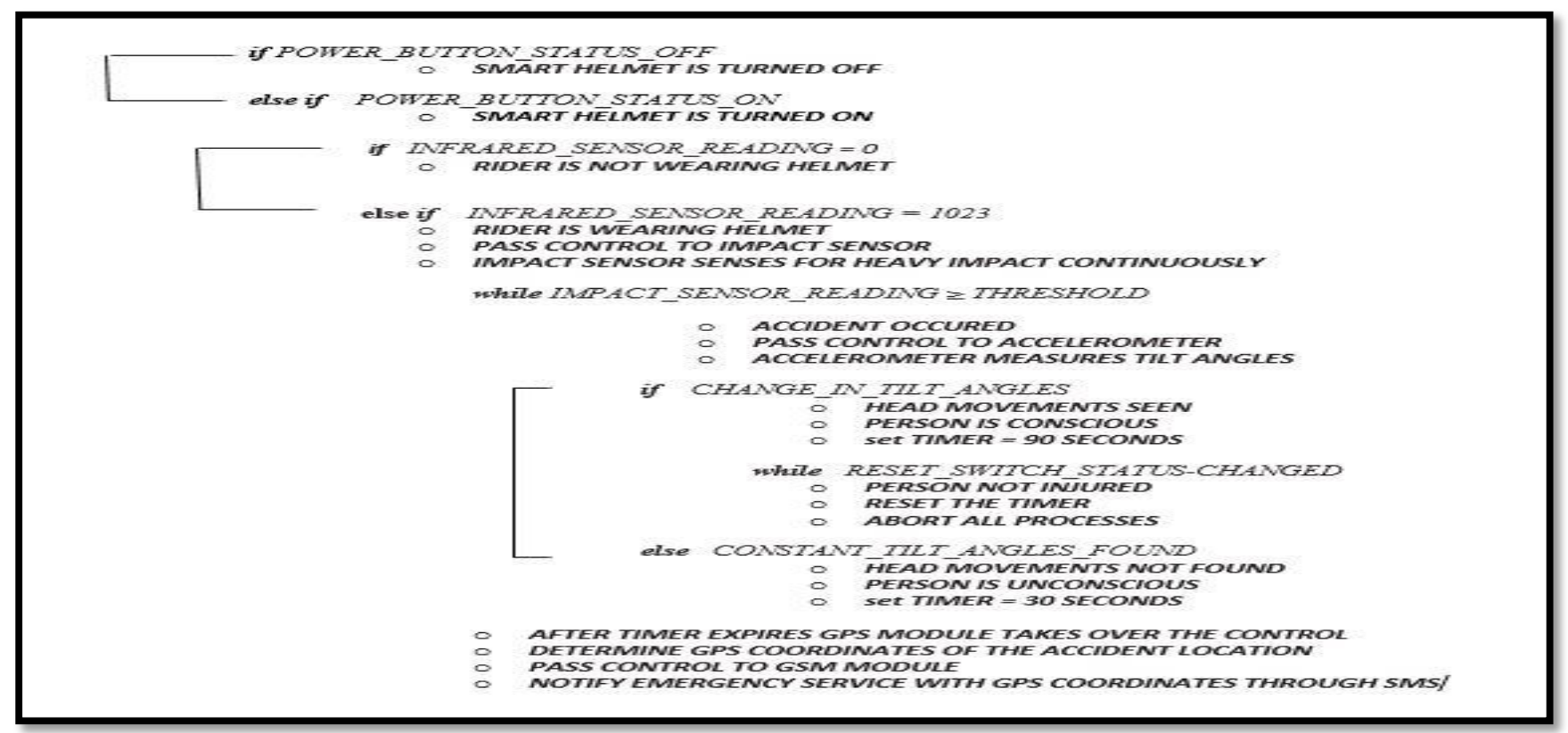

Algorithm 1. SHENS working algorithm

The algorithm takes numerous assumptions to function efficiently during real time. The assumptions that are strictly considered during the development of this algorithm are as follows: i) POWER_BUTTON_STATUS variable denotes the state of the power button which activates or turns off the smart helmet, ii) The infra-red sensor outputs the value 1023 into the variable INFRARED_SENSOR_READING if the obstacle, in this case, the head of the rider, is detected, iii) The infra-red sensor outputs the value 0 into the variable INFRARED_SENSOR_READING if the obstacle, in this case, the head of the rider, is not detected, iv) The variable THRESHOLD and its determination are discussed in Section 4 , v) The CHANGE_IN_TILT_ANGLES is the variation in the tilt angles that are calculated using (3), (4) and (5), which is mentioned in Section IV, vi) The
CONSTANT_TILT_ANGLES is the constant and steady tilt angles without any deviation, that are calculated using (3), (4) and (5) of Section IV, vii) The RESET_SWITCH_STATUS-CHANGED refers to the change in the state of the reset switch which can be used to reset the timer in case of no immediate medical assistance is needed.

\section{B. Components used in the Implementation of the Proposed} System

The proposed model makes use of a wide range of hardware to function to its fullest capacity. The various components that are used in developing this smart helmet are tabulated as shown in Table I.

\begin{tabular}{|l|l|l|}
\hline \multicolumn{1}{|c|}{ HARDWARE } & \multicolumn{1}{c|}{ PUBLE I } & SPECIFICATION \\
\hline Arduino Uno & To integrate and control the sensor network, GPS and GSM modules. & Microcontroller \\
\hline Infra-red sensor & To ensure the rider is wearing the helmet. & $\begin{array}{l}\text { Uses Obstacle detection } \\
\text { principle. }\end{array}$ \\
\hline Impact sensor & To determine the impact of the hit during the accident. & MEAS-SPEC sensor \\
\hline Accelerometer sensor & To detect the consciousness of the victim. & ADXL 335 \\
\hline 7 segment LED display & To indicate the timer countdown. & Anode Display \\
\hline GPS module & To determine the exact location of the accident. & Skylab 13BL \\
\hline GSM module & To establish communication with the emergency service. & SIM900A \\
\hline SIM Card & To send SMS to the emergency service. & Vodafone \\
\hline Batteries & To power the helmet and enhance portability. & 12 Volt 1 Amp Battery \\
\hline
\end{tabular}




\section{Interfacing with Arduino UNO}

The Smart Helmet with Emergency Notification System (SHENS) is implemented with the help of Arduino Uno, which is a programmable microcontroller capable of integrating various components like sensors, GSM and GPS modules. Arduino is an open-source prototyping platform for building embedded systems and electronic projects. Arduino also provides an Integrated Development Environment (IDE) that runs on a computer capable of uploading the compiled code into the physical Arduino board. The embedded C programming language is employed to program the sensors and other vital components. The prototypical connected system controlled by the Arduino is shown in Fig. 2.

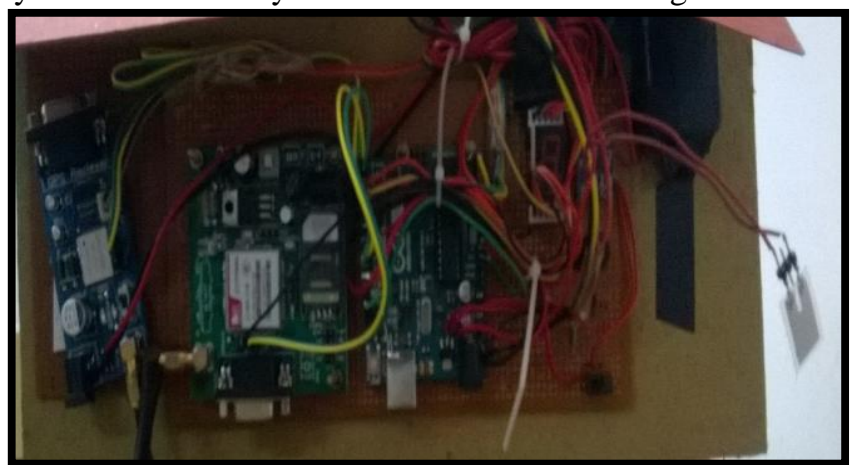

Figure 2. The connection between the Arduino Board and other components of SHENS

\section{Impact Determination using Impact Sensor}

The impact sensor detects the sudden impact on the surface of the helmet and outputs the analog value. The value should be converted into digital to derive the exact impact value of the hit. The actual impact a standard helmet can withstand is between the range $250 \mathrm{~g}-300 \mathrm{~g}$ as mentioned in the reference [6]. Even with this level of protection, concussions, broken neck, and head damage can happen. However, the same impact of over $100 \mathrm{~g}$ and above cannot be produced manually to simulate an accident scenario. Hence, in this work for the purpose of experimentation, the threshold impact is set to $2.5 \mathrm{~g}$ for the convenience of testing, upon which the helmet reacts.

The impact of the hit on the surface of the helmet can be derived using the following key points. The first one, the analog default range of the impact sensor is 0 to 1023 and the digital default range of the impact sensor is between 0 volts and 5 volts.

In order to convert the analog values from the impact sensor into digital values the equation Eq. 1 given below is used.

$$
\frac{\text { Raw Analog value from the sensor }}{\text { Maximum number of analog states }}=\frac{\text { Voltage }}{\text { of the sensor }}
$$

The g-force or acceleration due to gravity acting on the object resting on the surface of the earth is $1 \mathrm{~g}$. The maximum number of analog states of the impact sensor is 1023 by default. The reference value used is $3.3 \mathrm{~V}$ used in the Arduino board for the implementation purposes.

Therefore using Eq.2,

$$
\text { Impact of the hit }=\text { Voltage }
$$

Hence it can be understood that the digital voltage is directly proportional to the acceleration due to gravity or the impact of the hit.

\section{E. Consciousness Detection using Accelerometer}

The consciousness of the accident victim can be analyzed using the accelerometer sensor. The accelerometer sensor has a default range of $\pm 3 \mathrm{~g}$. The accelerometer sensor outputs a series of $\mathrm{X}, \mathrm{Y}$ and $\mathrm{Z}$ values which are analog values. These values are converted to digital which in turn converted into angles. The readings are monitored for a few microseconds. The tilt angle of the helmet is calculated using the analog values from the accelerometer with the following formula.

$\mathrm{X}, \mathrm{Y}$ and $\mathrm{Z}$ are the analog values from the accelerometer. The corresponding angles AX, AY and AZ are calculated using the arctan function which is presented in Eq. 3, Eq. 4 and Eq. 5 below.

$$
\begin{aligned}
& \mathrm{AX}=\arctan \left(\mathrm{X} / \sqrt{ }\left(\mathrm{Y}^{\wedge} 2+\mathrm{Z}^{\wedge} 2\right)\right. \\
& \mathrm{AY}=\arctan \left(\mathrm{Y} / \sqrt{ }\left(\mathrm{X}^{\wedge} 2+\mathrm{Z}^{\wedge} 2\right)\right. \\
& \mathrm{AZ}=\arctan \left(\mathrm{Z} / \sqrt{ }\left(\mathrm{X}^{\wedge} 2+\mathrm{Y}^{\wedge} 2\right)\right.
\end{aligned}
$$

The person can be said to be conscious or unconscious based on the following observations by the accelerometer sensor. If there are deviations in the angles sensed by the accelerometer, the victim is expected to have head movements ensuring that he or she is conscious. If the tilt angles are same for a certain amount of time, then it is understood that the person is unconscious. Thus, accelerometer sensor is employed in SHENS to determine the degree of consciousness of the person.

Finally, the program to carry out all the essential activities of the smart helmet is typed in the Arduino IDE and compiled. The successfully compiled program is uploaded into the board to initiate the effective functioning of the helmet.

\section{RESULTS}

The internal working of SHENS can be well understood by having a look at the Arduino console. The screenshots below explain the step by step outputs of the various integrated modules of SHENS. The impact values from the impact sensor can be presented in a graph in order to easily visualize the deviations over time. The graph indicating the determination of threshold calculated using (1) and (2) of the implementation section is presented below in Fig. 3.

After the threshold point of $2.5 \mathrm{~g}$ which is set for the ease of testing, the control is transferred to the accelerometer sensor to determine the consciousness of the victim. Fig. 4 is the output of the internal working of the SHENS when the 
accident victim is conscious. The deviations in the angles obtained with the help of accelerometer sensor shows that the victim is found to have constant head movements indicating that the victim has not lost his/her consciousness. This deviation can be clearly seen in the graph shown in Fig. 5.

Fig. 6 shows the output from the serial monitor of the Arduino showing the operations carried out by the SHENS in the case when the person is unconscious. The absence of deviations in the angles obtained with the help of accelerometer sensor shows that the victim is not found to have head movements indicating that the victim has lost his/her consciousness. This deviation can be clearly seen in the graph depicted in Fig. 7.

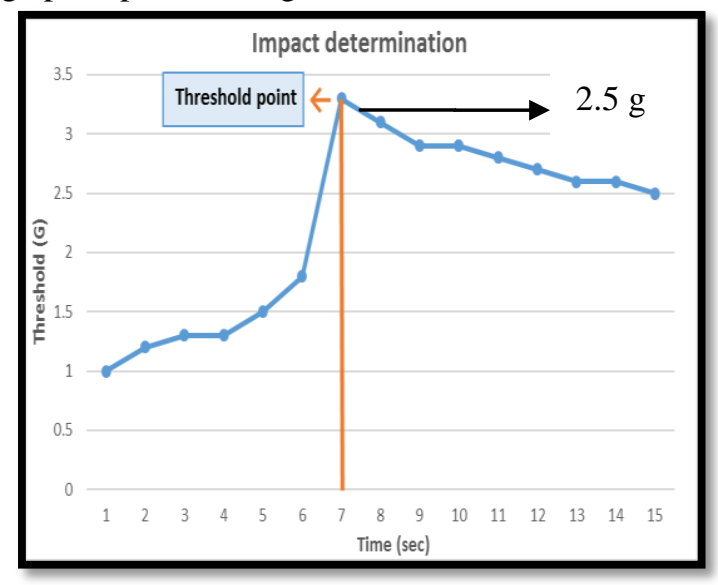

Figure 3. Visualization of impact determination

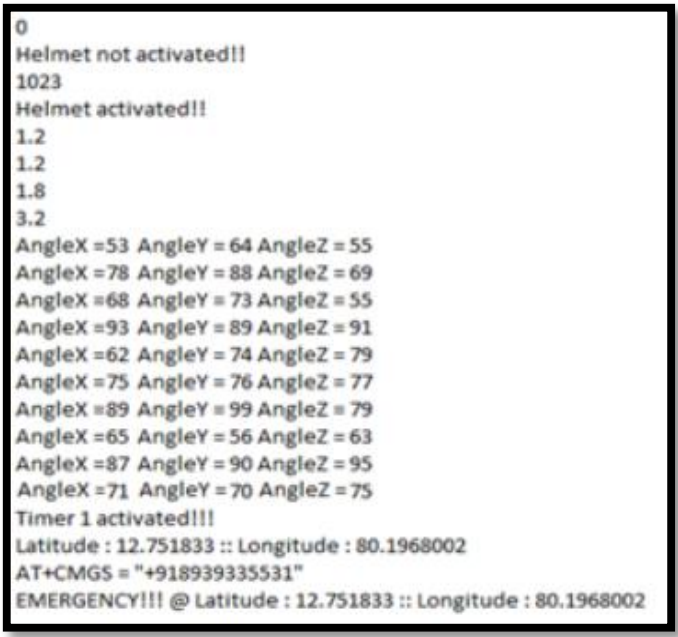

Figure 4. Serial monitor output when the victim is conscious

After the accelerometer sensor determines whether the victim is conscious or unconscious, the need to initiate emergency notification arises. The timer gets set for 90 seconds, if the victim is conscious and for 30 seconds if the person is unconscious. After the timer expires, the GPS module determines the location of the accident. The GPS coordinates are sent to the emergency contact as an SMS using the GSM module.

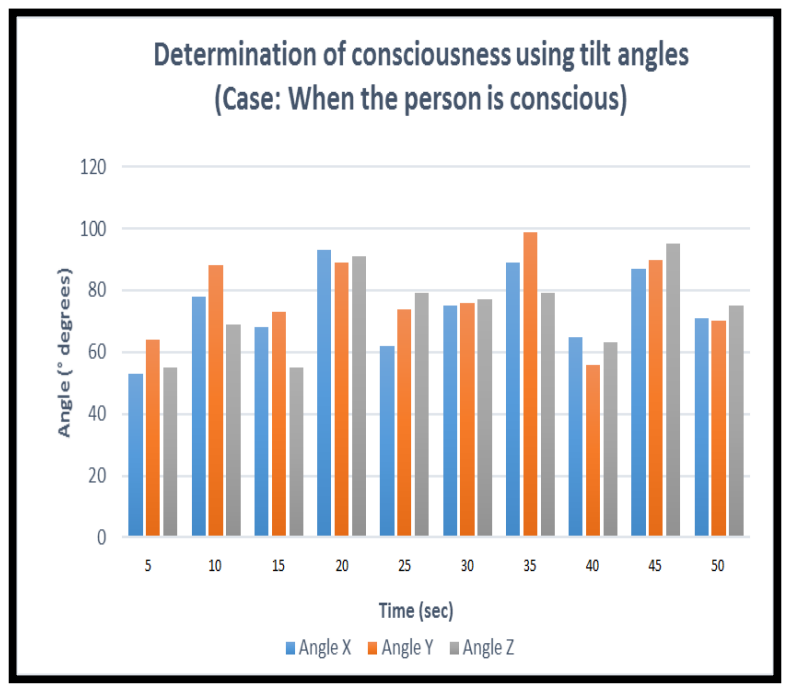

Figure 5. Graphical deviations in the tilt angles when the person is conscious

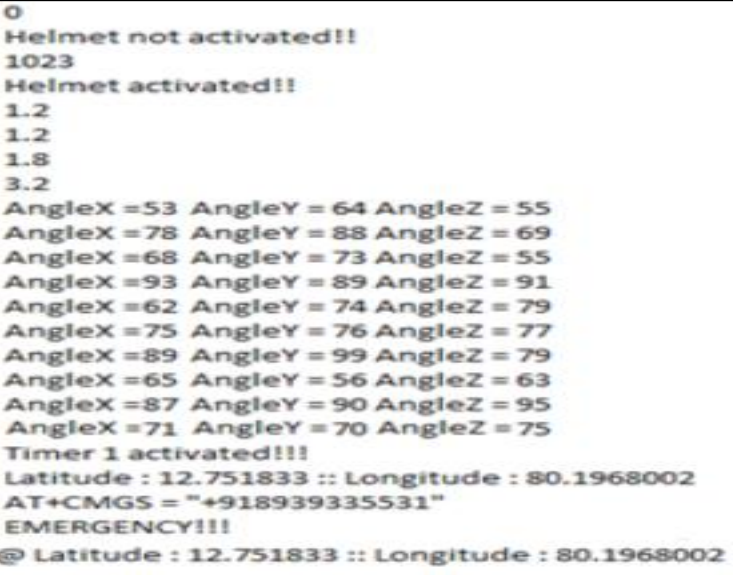

Figure 6. Serial monitor output when the victim is unconscious

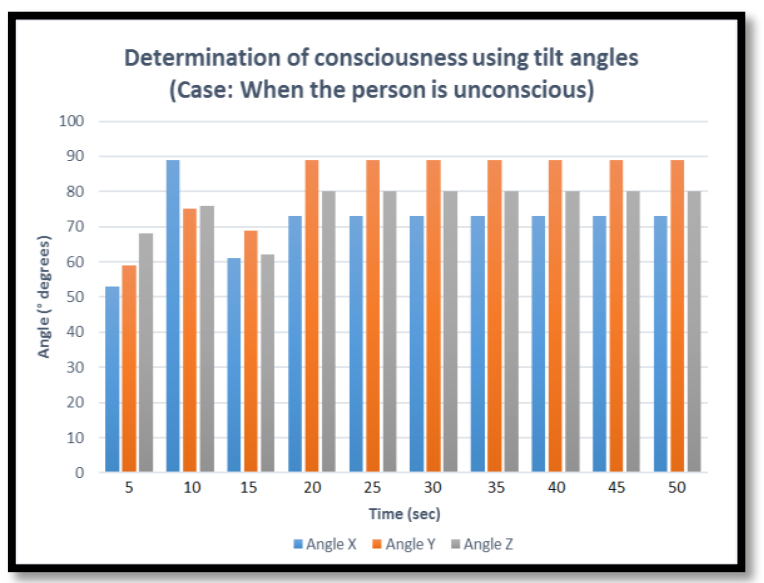

Figure 7. Graphical deviations in the tilt angles when the person is unconscious 


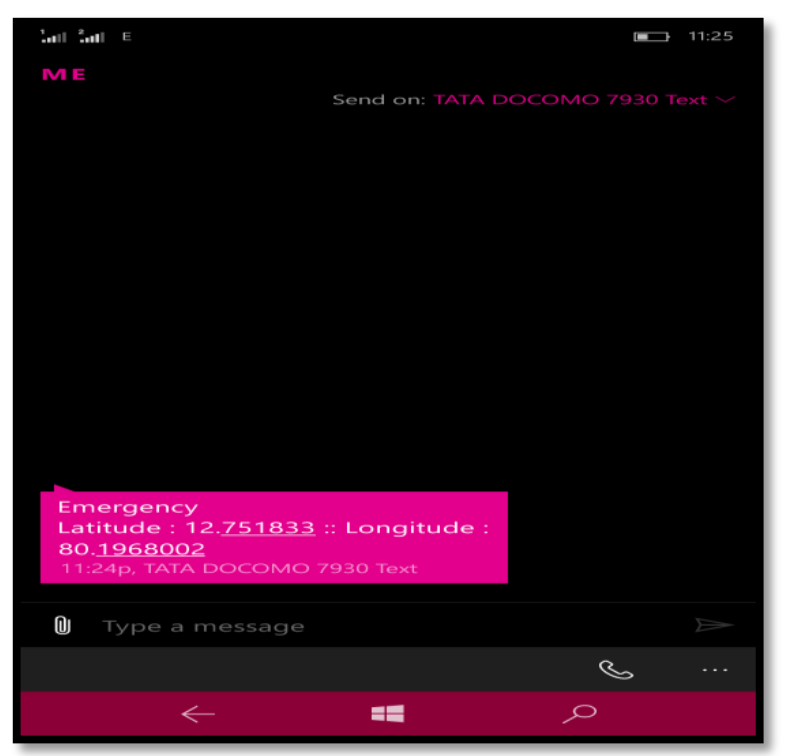

Figure 8. SMS from SHENS during the experimental accident

The SMS containing the GPS coordinates is sent to the intended emergency number which is already preprogrammed as soon as the timer gets expired. Fig. 8 indicates the SMS from SHENS during the experimental accident.

\section{CONCLUSION AND FUTURE WORK}

The SHENS has the scope to become a daily gadget like a mobile phone since it has the potential to save people from the danger of losing their lives during road travel. A large number of people using two wheelers for their day to day chores can make use of SHENS ensure their safety during road travel. This helmet can be very much helpful when the accidents happen in the highways and scarcely crowded roads as the Smart Helmet with Emergency Notification System (SHENS) has the potential to help the accident victims to get timely medical assistance without any interventions from anyone around. There are some improvements and feature addition that can be done to SHENS in order to provide the humanity a better user experience. Some of the works that can be done in future are as follows. An emergency push button that can send the notification directly to the emergency service in case of the helmet not experiencing a threshold impact but accident victim is badly hurt. An alcohol detection sensor which can detect alcohol in the breath of the rider can be added to the system in order to send text alerts indicating drunken drive by the rider to the nearest police station by integrating with the GSM module.

\section{ACKNOWLEDGMENT}

This research was financially supported by the management of SSN College of Engineering, Chennai Tamilnadu, India.

\section{REFERENCES}

[1] Article from www.knowindia.net online available from http://www.knowindia.net/auto.html.

[2] Gubbi, J., Buyya, R., Marusic, S., and Palaniswami, M. Internet of things (IoT): A vision, architectural elements, and future directions. Future Gener. Comput. Syst. 29, 7, pp. 1645-1660, Sept. 2013.

[3] R. Sujatha, N. VijayaRagavan, K.S. Suganya, "IOT: To enhance automatic accident notification using M2M technologies", International Journal of Scientific \& Engineering Research, Vol. 6, No. 3, pp. 1-4, March 2015.

[4] Fogue M, Garrido P, Martinez FJ, Cano JC, Calafate CT, Manzoni P, "A System for Automatic Notification and Severity Estimation of Automotive Accidents", IEEE Transactions on Mobile Computing, Vol. 13, No. 5, pp. 948 - 963, May 2001.

[5] "Traffic Accident Automatic Detection And Remote Alarm Device" Wang Wei and Fan Hanbo

[6] Article from the Wikipedia online dated 2015 hosted online available from https://en.wikipedia.org/wiki/Transport_in_India\#Two_Wheeler.

[7] Article on Helmets.org online available on March 7, 2015 from http://www.helmets.org/ideal.htm.

[8] "Fatality Analysis Reporting System General Estimate System - 2011 Data summry" - United States Department of Transportation.

[9] K. Sudarsan, P. Kumaraguru Diderot, "Helmet for Road Hazard Warning with Wireless Bike Authentication and Traffic Adaptive Mp3 Playback", International Journal of Science and Research (IJSR), Vol. 3, No. 3, March 2014.

[10] L Huan-liang, Q Hong-mei, F Ke, L Dong-lin, "Design of Human computer interaction helmet for wearable computer", IEEE International Conference on E -Business and E -Government (ICEE), pp. 1-4, May 2011.

[11] IR Sensor - http://education.rec.ri.cmu.edu/content/electronics/ boe/ir_sensor/1.html.

[12] Impact Sensor http://electronicdesign.com/analog/low-cost-impact-sensor-usespiezoelectric-device.

[13] MEMS - http://shockwatch.com/products/impact-and-tilt.

[14] Accelerometer Sensor https://www.bosch-sensortec.com/bst/products/motion/ accelerometers/overview_accelerometers.

[15] Arduino - https://www.arduino.cc/.

[16] GPS with arduino http://www.instructables.com/id/Connecting-GPS-moduleto-Arduino/.

[17] GSM with https://www.arduino.cc/en/Guide/ArduinoGSMShield. arduino- 